\title{
The Therapeutic Potential of Glutathione Supplement: A Review of Clinical Trials
}

\author{
Abdelrahim Hunaiti
}

\begin{abstract}
Glutathione (GSH) is a tripeptide ( $\gamma$-glutamyl cysteinyl glycine) involved in a variety of biological processes indispensable to sustain life and the most abundant free radical scavenger synthesized endogenously in humans. There are adverse health consequences from glutathione deficiency. The present mini-review aims to provide an extensive overview to glutathione supplement therapeutic effects in human subjects.
\end{abstract}

Index Terms - Glutathione; Therapeutic Supplementation; Free radical scavenger.

\section{INTRODUCTION}

Glutathione (GSH) is an antioxidant present in plants, animals, fungi, and some bacteria and the most important antioxidant found in mammalian cells. structurally glutathione is a tripeptide consisting of glutamic acid attached via its side chain to the N-terminus of cysteinylglycine to synthesis GSH, $(\gamma$-L-glutamyl-Lcysteinylglycine). Due to the sulfhydryl group of cysteine it can exists in either reduced (GSH) or oxidized (GSSG) form. Because of its regulatory functions against oxidative stress and maintaining thiol homeostasis, $90 \%$ of glutathione under normal circumstances are in a reduced form $(\mathrm{GSH})$ while in abnormal condition of oxidative stress, glutathione concentration in oxidized form (GSSG) is more abundant $[1,2]$. The GSH: GSSG ratio is the primary determinant of the cellular redox state and the processes of glutathione synthesis, transport, utilization, and metabolism are tightly controlled to maintain intracellular glutathione homeostasis and redox balance. An elevation in the GSSG content and decreases in GSH content are indicative of an increases production of free radicals and ultimately lead to systemic oxidative stress and the risk of acquiring serious and lifethreatening diseases [2]. Many studies indicate that disturbances in cellular glutathione homeostasis and redox status are implicated in the etiology and/or progression of a number of acute and chronic human diseases, including neurodegenerative diseases , cancer, cystic fibrosis, cardiovascular, inflammatory, immune, metabolic, and aging diseases $[3,4,5,6]$. Supplementation with the glutathione precursor's cysteine and glycine fully restores glutathione synthesis and lowers levels of oxidative stress and oxidant damages [7, 8]. However, supplementing with glutathione precursors relies upon the body's ability to synthesize glutathione from these precursors. Supplementing GSH for people at risk for glutathione redox

Published on April 09, 2020.

Abdelrahim Hunaiti, Department of Clinical Laboratory Sciences, School of Science, University of Jordan, Jordan.

(e-mail: author@boulder.nist.gov) balance has limited due to rapid breakdown during oral ingestion. Allen J, and Bradley RD [9] studied the effects of oral glutathione supplementation on systemic oxidative stress biomarkers in forty adult volunteers without acute or chronic disease participated in their study. They reported no significant changes in biomarkers of oxidative stress, including GSH status, GSSG, and ratio GSH: GSSG in the human volunteers. While Richie JP et al [10] in their 6month randomized, double-blinded, placebo-controlled trial of supplementation oral GSH (250 or $1,000 \mathrm{mg} /$ day $)$ showed, for the first time, that daily consumption of GSH supplements was effective at increasing body stores of glutathione. Similarly Sinha, R. et al [11] showed that Oral supplementation with liposomal glutathione elevates body stores of glutathione and markers of immune function. Schmitt, B et al. [12] in their study demonstrated the significant superiority of a new sublingual form of GSH over the oral form, both in terms of bioavailability and positive effects on oxidative stress. Recent study in our lab highlighted the Clinical uses of bilingual glutathione supplementation as a novel treatment for cystine stone [13] our results showed that patients who took glutathione over 3 months had no new stone formation and the size of the already presented stones was still the same or reduced, while those patients who took placebo for the same period developed new stones and the size of already presented stones increased. Overwhelming evidence shows that maintaining glutathione levels may help with the symptoms of neurodegenerative diseases. Brain glutathione level was suggested as a biomarker for mild cognitive impairment and Alzheimer's disease and elevated glutathione as a therapeutic strategy in Alzheimer's disease and Cognitive Improvement with Glutathione Supplement [14,15 ,16,17,18]. Their findings appear to support glutathione as a potential therapeutic agent. Kern JK et al [19] found that oral glutathione supplements or injections might reduce some effects of Autism spectrum disorders (ASD) AlOmari et al [20] reported that oxidative stress played a crucial role in the wide spread of ASD in Jordan in combination with, the C677T polymorphism of MTHFR gene in the etiology of the disorder and that Oral sublingual -glutathione given to autistic children improved their deterioration of GSH homeostasis and oxidative stress as well as some of their behavioral symptoms such as cooperation, imitation, play, leisure and social interaction.

\section{GLUTATHIONE AND ITS ANTI-AGING AND SKIN WHITING EFFECTS}

Another major effect of Glutathione imbalance in the body is premature aging.

The functional application and therapeutic potential of 
glutathione in the treatment of aging-associated diseases and the antimelanogenic properties of glutathione are well documented in the literature $[21,22]$.It is well known that Glutathione deficiency in elderly humans occurs because of a marked reduction in glutathione synthesis and dietary supplementation with the glutathione precursors cysteine and glycine fully restores glutathione synthesis and concentrations and lowers levels of oxidative stress and oxidant damages. The antimelanogenic properties of glutathione is due to its ability to scavenge ultraviolet radiation induced reactive oxygen species generated in epidermal cells and blocking the induction of tyrosinase the enzyme essential for the skin pigment melanin by these peroxides [22] these findings has led to its promotion as a skin-lightening agent. GSSG was preferred over GSH, as GSH is unstable in aqueous solutions. GSSG eventually generates GSH after cutaneous absorption [23, 24].

\section{CONCLUSION}

Glutathione is generally a safe ingredient for use and is regarded as food or health supplements in most countries, while it is considered a pharmaceutical agent in some others. Could be used both as a protective and therapeutic agent against several diseases. Despite the therapeutic benefits of glutathione, the major challenge is attributed to its poor bioavailability due to rapid breakdown during oral ingestion the bioavailability can be enhanced through several innovative ways. We feel that this product has the potential to do a great deal of good for a large number of people

\section{REFERENCES}

[1] Aquilano, K., Baldelli, S., \& Ciriolo, M. R. (2014). Glutathione: new roles in redox signaling for an old antioxidant. Frontiers in pharmacology, $5 ; 196$.

[2] He,L He,T. Farrar,S. Ji,L. Liu,T and Ma,X ( 2017) "Antioxidants maintain cellular redox homeostasis by elimination of reactive oxygen species," Cellular Physiology and Biochemistry, vol. 44, no. 2, pp. 532-553.

[3] Castegna A, Lauderback C, Drake J. (2009) Oxidative stress and neurodegenerative diseases: A review of upstream and downstream antioxidant therapeutic options. Current Neuropharmacology. 7(1):6574.

[4] Bajic VP, Van Neste C, Obradovic M, Zafirovic S, Radak D, Bajic VB, et al.( . 2019) Glutathione "redox homeostasis" and its relation to cardiovascular disease. Oxidative Medicine and Cellular Longevity.1-14.

[5] Ballatori N, Krana SM,Notenboom S, Shi S. Tien K, Hammont CL. (2009) Glutathione dysregulation and the etiology and progression of human diseases.Biol.Chem;39(3):191-214.

[6] Liu, Z., Zhou, T., Ziegler, A. C., Dimitrion, P., and Zuo, L. (2017). Oxidative stress in neurodegenerative diseases: from molecular mechanisms to clinical applications. Oxid. Med. Cell. Longev.4:1-11.

[7] Sekhar, R.V.; Patel, S.G.; Guthikonda, A.P.; Reid, M.; Balasubramanyam, A.; Taffet, G.E.; Jahoor, F. (2011) Deficient synthesis of glutathione underlies oxidative stress in aging and can be corrected by dietary cysteine and glycine supplementation. Am. J. Clin. Nutr. 94, 847-853.

[8] Rushworth, G.F.; Megson, I.L.( 2014) Existing and potential therapeutic uses for $\mathrm{N}$-acetylcysteine: the need for conversion to intracellular glutathione for antioxidant benefits. Pharmacol. Ther., $141,150-159$.

[9] Allen J, Bradley RD.(2011) Effects of oral glutathione supplementation on systemic oxidative stress biomarkers in human volunteers. J Altern Complement Med. (9); 827-833.

[10] Richie, J.P., Jr.; Nichenametla, S.; Neidig, W.; Calcagnotto, A.; Haley, J.S.; Schell, T.D.; Muscat, J.E. (2015) Randomized controlled trial of oral glutathione supplementation on body stores of glutathione. Eur. J. Nutr., 54, 251-263.

[11] Sinha, R.; Sinha, I.; Calcagnotto, A.; Trushin, N.; Haley, J.S.; Schell, T.D.; Richie, J.P., Jr. Oral supplementation with liposomal glutathione elevates body stores of glutathione and markers of immune function. Eur. J. Clin. Nutr. 2018, 72, 105-111.

[12] Schmitt B, Vicenzi M, Garrel C, Denis FM (2015). Effects of Nacetylcysteine, oral glutathione (GSH) and a novel sublingual form of GSH on oxidative stress markers: A comparative crossover study. Redox Biology. 6:198-205.

[13] Al-Shehabat, M.., Hani, I., Jaradat, S.. Alaraji ,A, and Hunaiti,A.(2017) Glutathione is a novel treatment for cystine stones.Comp Clin Pathol. 26, (3) 697-705

[14] Casetta I , Govoni V , Granieri E (2005) Oxidative stress, antioxidants and neurodegenerative diseases. Curr Pharm Design 11, 2033-2052.

[15] Cummings JL (2004) Drug therapy - Alzheimer's disease. N Engl J Med 351, 56-67

[16] Mandal PK , Saharan S , Tripathi M , Murari G (2015) Brain glutathione levels-a novel biomarker for mild cognitive impairment and Alzheimer's disease. Biol Psychiatry 78, 702-710.

[17] Butterfield DA, Pocernich CB , Drake J (2002) Elevated glutathione as a therapeutic strategy in Alzheimer's disease. Drug Dev Res 56, 428-437.

[18] Mandal, Pravat \& Shukla, Deepika \& Tripathi, Manjari \& Ersland, Lars. (2019). Cognitive Improvement with Glutathione Supplement in Alzheimer's Disease: A Way Forward. Journal of Alzheimer's disease: JAD. 68. 531-535. 10.3233/JAD-181054

[19] Kern JK, Geier DA, Adams JB, Garver CR, Audhya T, Geier MR.( 2011) A clinical trial of glutathione supplementation in autism spectrum disorders. Med. Sci. Monit. 17(12): 677-682

[20] Al-Omari,L , Abdelrahim A. Hunaiti, Mohammad A. Beirat and Bustanji,Y. (2019) Study of MTHFR C677T Polymorphism and Associated Oxidative Stress Biomarkers among Autism Spectrum Disorder Patients in Jordan. Jordan Journal of Biological Science (JJBS); Accepted August 29, 2019.will be published in Volume 13, Number 3 (September), 2020.

[21] Homma T, Fuji i J. (2015) Application of Glutathione as AntiOxidative and Anti-Aging Drugs. Curr Drug Metab. 16(7):560-71.

[22] Weschawalit, Sinee \& Thongthip, Siriwan \& Phutrakool, Phanupong \& Asawanonda, Pravit. (2017). Glutathione and its antiaging and antimelanogenic effects. Clinical, Cosmetic and Investigational Dermatology. Volume 10. 147-153

[23] Villarama CD, Maibach HI. (2005) Glutathione as a depigmenting agent: An overview. Int J Cosmet Sci.27:147-53

[24] Watanabe F, Hashizume E, Chan GP, Kamimura A. ( 2014) Skinwhitening and skin-condition-improving effects of topical oxidized glutathione: a double-blind and placebo-controlled clinical trial in healthy women. Clin Cosmet Investig Dermatol.7:267-274. 